\title{
Die Wirkung von hellroter und blauer Strahlung auf die Chloroplastenausbildung
}

\author{
Von Rainer Bergfeld \\ Aus dem Botanischen Institut der Universität Freiburg i. Br. \\ (Z. Naturforschg. 18 b, 328-331 [1963]; eingegangen am 15. Oktober 1962)
}

\begin{abstract}
Growth and morphogenesis of the young fern gametophytes, e. g. Dryopteris filix-mas, is completely controlled by light. This remarkable light effect has nothing to do with photosynthesis but is a good example of photomorphogenesis, i. e. direct control of growth and development by light (Монr und Оhlenroth) ${ }^{1}$. - Only short-wave-length visible light ("blue light") has been found to the photomorphogenically effective. Under longer wave length visible light ("red light") the sporelings continue to grow as "etiolated" filaments very similar to those formed in darkness, while under blue light "normal" two dimensional growth and prothallium formation can occur.

The induction of morphogenesis by blue light is causally connected with an increase of protein synthesis. Sporelings growing in the blue contain much more protein (as related to dry weight) than sporelings growing in the red at about the same photosynthetic rate (OHLEnroth und Мohr) ${ }^{2}$.

In the present paper it has been shown that the size of the chloroplasts in cells of these sporelings can be controlled by light. In darkness the chloroplasts are smaller than in red or blue but in blue they become much larger than in the red. This control of plastid size by light is reversible, e. $g$. if sporelings which have been grown in the red are kept under blue light the chloroplast size increases rapidly up to a certain value but if they are brought back into the red the original size will be regained within a few days.

It has been concluded that the protein content of the plastids is controlled by light and that changes of the plastid size are manifestations of a change in protein content of the plastids.
\end{abstract}

Die Morphogenese der Vorkeime von Dryopteris filix-mas, d.h. die Entwicklung des zwei- bis dreidimensionalen Prothalliums aus dem fädigen Protonema, wird durch Blaulicht gesteuert $(\text { Монг })^{3}$. Der Photosynthese kommt hierbei keine Bedeutung zu (Mohr und Оhlenroth ${ }^{1}$. Die Morphogenese ist mit einer Erhöhung des Proteingehaltes verbunden (Hotta und Osawa ${ }^{4}$, Ohlenroth und Mohr ${ }^{2}$ ) sowie mit Chloroplasten-Wachstum und ChloroplastenVermehrung $\left(\mathrm{MoHr}^{3}\right)$. Es liegt nahe, eine Beziehung zwischen der Chloroplasten-Ausbildung und Proteinsynthese anzunehmen, zumal Brawermann et al. ${ }^{5}$, Zucker und Stinson JR. ${ }^{6}$ und andere nachwiesen, daß in grünen Zellen die Proteine überwiegend in den Chloroplasten gelagert sind (vgl. auch HE$\mathrm{BER}^{7}$ ). In der vorliegenden Arbeit wird die Entwicklung der Chloroplasten unter verschiedenen Lichtqualitäten verfolgt, unter Berücksichtigung der morphogenetischen Wirkung des applizierten Lichtes und der damit verbundenen Umsteuerung des Zellstoff wechsels.

1 H. Мohr u. K. Ohlenroth, Planta 57, 656 [1962].

2 K. Оhlenroth u. H. Mohr, Planta 1962 (i. p.).

${ }^{3}$ H. Mohr, Planta 47, 127 [1956].

4 Y. Нотta u. S. Osawa, Exp. Cell Res. 15, 85 [1958].

\section{Experimentelles}

Das verwendete Sporenmaterial (vgl. Mонr und Ohlenroth ${ }^{1}$ ) wurde $1959 \mathrm{im}$ Tübinger Botanischen Garten gesammelt und von Sporangienresten gereinigt. Es wird seitdem bei $6{ }^{\circ} \mathrm{C}$ aufbewahrt. Die Keimfähigkeit beträgt immer noch praktisch 100 Prozent. Die Aussaat erfolgte in Schälchen von $5 \mathrm{~cm}$ Durchmesser und $2 \mathrm{~cm}$ Höhe, die mit $7 \mathrm{ml}$ einer 1,5-proz. Agarlösung ausgegossen waren. Gelöst wurde der Agar in folgender Nährlösung: $0,2 \mathrm{~g} \quad \mathrm{NH}_{4} \mathrm{NO}_{3} ; 0,1 \mathrm{~g} \quad \mathrm{~K}_{2} \mathrm{HPO}_{4} ; 0,1 \mathrm{~g}$ $\mathrm{MgSO}_{4} \cdot 7 \mathrm{H}_{2} \mathrm{O} ; 0,1 \mathrm{~g} \mathrm{CaCl}_{2} ; 1-2$ Tropfen 1-proz. $\mathrm{FeCl}_{3}$ auf $1000 \mathrm{ml}$ dest. Wasser. Der Ausgangs- $p_{\mathrm{H}}-$ Wert betrug 6,0 $\pm 0,2$. Um die Schalen vor dem Austrocknen zu schützen, wurden sie mit einer Glasplatte bedeckt und mittels Wollfett abgedichtet. Die Keimungsinduktion erfolgte durch $72 \mathrm{Stdn}$. Hellrotbestrahlung (Standardfeld). Danach kamen die Schalen weitere 72 Stdn. ins Dunkle, um ein gleichmäßiges Keimen zu erreichen. Erst nach dieser Vorbehandlung folgte das Einsetzen in die Lichtfelder bzw. ins Dunkle. Dieser Zeitpunkt gilt als Versuchsbeginn (= Keimung).

Die Durchführung der Versuche erfolgte in Konstanträumen bei Dauerlicht. Die Temperatur betrug auf der Tischplatte, auf der die Schalen standen, 20,0 $\pm 0,5{ }^{\circ} \mathrm{C}$.

\footnotetext{
5 G. Brawermann, A. O. Pogo u. E. Chargaff, Biochim. biophysica Acta [Amsterdam] 48, 418 [1961].

G M. Zucker u. H. T. Stinson JR., Arch. Biochem. Biophysics 96, 637 [1962].

7 U. Heber, Nature [London] 195, 91 [1962].
} 
Als Lichtquellen dienten folgende Leuchtstoffröhren: Hellrot Osram TL 40 W/15; Blau Osram TL W/18. Die Aggregate waren zur Sicherung gegen Licht unerwünschter Wellenlängen zusätzlich noch mit einer roten oder blauen Plexiglasscheibe (Fa. Röhm und Haas, Darmstadt) versehen. Das Hellrotfeld hatte sein Emis sionsmaximum bei $660 \mathrm{~m} \mu$, wobei ein Band von 610 bis $700 \mathrm{~m} \mu$ durchgelassen wurde. Das Blaulicht besa das Maximum bei $445 \mathrm{~m} \mu$ und die verwendete Plexiglasscheibe bei $400 \mathrm{~m} \mu$ und bei $520 \mathrm{~m} \mu$ eine Durchlässigkeit von 2,5 Prozent. Das Hellrot- und das Blaulichtfeld waren möglichst genau auf gleiche Quantenstromdichte eingestellt. Die Intensitäten nahmen während der Versuchszeit im Blaufeld von 760 auf $660 \mathrm{erg} / \mathrm{cm}^{2} \mathrm{sec}$ und im Hellrotfeld von 580 auf $520 \mathrm{erg} / \mathrm{cm}^{2} \mathrm{sec}$ ab. Bei der Intensitätsangabe ist zu beachten, daß die zur Messung verwendeten Thermosäulen einen großen Cosinusfehler besitzen. - Die verwendeten Bestrahlungsanlagen und Meßgeräte sind bei Mohr und Pinnig $^{8}$ näher beschrieben.

Die Versuchsdauer betrug nicht mehr als 28 Tage, vor allem, weil bei den im Blaulicht gehaltenen Schalen der $p_{\mathrm{H}}$-Wert des Agars stark abnahm (bis auf $p_{\mathrm{H}} 4,8$ ). Die Versuche wurden in folgenden Kombinationen durchgeführt * :

a) Dauer-HR; Dauer-Bl; Dauer-DK.

b) Einfacher Umsetzversuch: $\mathrm{HR} \rightarrow \mathrm{Bl}$; $\mathrm{Bl} \rightarrow \mathrm{HR}$; $\mathrm{Bl} \rightarrow \mathrm{DK} ; \mathrm{DK} \rightarrow \mathrm{Bl} ; \mathrm{DK} \rightarrow \mathrm{HR}$; wobei der Zeitpunkt des Umsetzens 7, 14 oder 21 Tage nach der "Keimung" lag.

c) Doppelter Umsetzversuch: $\mathrm{HR} \rightarrow \mathrm{Bl} \rightarrow \mathrm{HR}$; DK $\rightarrow$ $\mathrm{Bl} \rightarrow \mathrm{DK}$; die zeitlichen Kombinationen ergeben sich aus Abb. 4 und 5.

Die Messungen erfolgten nur an Chloroplasten der Basalzelle, weil diese Zelle sieben Tage nach Versuchsbeginn voll entwickelt ist und nicht mehr weiter wächst. Die Basalzelle ist die erste ausgebildete Zelle des Vorkeimes. Es wurde jeweils der größte Plastidendurchmesser mittels eines Okularmikrometers bei 1600-facher Vergrößerung bestimmt. Jeder Meßpunkt einer Kurve entspricht dem Mittelwert von 250 (5 Parallelversuche) bei den DK-Versuchen bzw. bei allen anderen Versuchen von 400 (8 Parallelversuche) Messungen. Der mittlere Fehler $\Delta D$ des arithmetischen Mittels der Durchmesser wurde nach der üblichen Formel z. B.

$$
\Delta D= \pm \sqrt{\frac{\left(D_{\mathrm{m}}-D\right)^{2}}{N \cdot(N-1)}}
$$

errechnet. Zwei Werte von $D$ werden als signifikant verschieden betrachtet, wenn die Differenz größer als der dreifache mittlere Fehler ist.

8 H. Mohr u. E. Pinnig, Planta 58, 569 [1962].

* Es werden folgende Abkürzungen verwendet: Hellrotlicht $=\mathrm{HR} ;$ Blaulicht $=\mathrm{Bl} ;$ Dunkel $=\mathrm{DK}$.

\section{Experimentelle Ergebnisse}

a) Die Entwicklung der Chloroplasten im HR, Bl und DK

Die Versuche zeigen, daß die Größe der sich bildenden Chloroplasten in den Vorkeimen von Dryopteris filix-mas von der Wellenlänge des applizierten Lichtes abhängig ist. Die Chloroplasten entwikkeln sich unter gleichbleibender Lichtqualität und Lichtintensität bis zu einer bestimmten Größe und behalten diese bei. Die Plastiden in den DK-Vorkeimen (denjenigen Vorkeimen, die nur zur Keimungsinduktion HR ausgesetzt waren) sind meist ungefärbt und ihr Durchmesser beträgt im Durchschnitt $3 \mu$, bei einem mittleren Fehler von nahezu $15 \%$ (Abb. 1). Die Chloroplasten in den Zellen der HR-

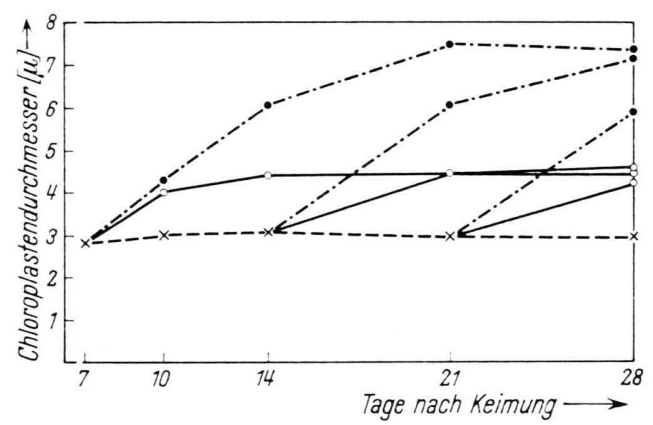

Abb. 1. Die Plastidengröße in den DK-Kontrollen und ihre Zunahme nach Umsetzen in $\mathrm{Bl}$ und $\mathrm{HR}$. $(----\times) \mathrm{DK}=$ Dunkel, $(-\cdot-\cdot-\cdot \bullet) \mathrm{Bl}=$ Blaulicht, $(-\mathrm{C}) \mathrm{HR}=$ Hellrotlicht.

Vorkeime liegen wandständig. Diese erreichen 14 Tage nach der Keimung einen maximalen Durchmesser von 4,8 $\pm 0,2 \mu$ (Abb. 2). Ähnlich liegen die Verhältnisse in den Zellen der Bl-Vorkeime, nur erreichen hier die Chloroplasten einen Durchmesser

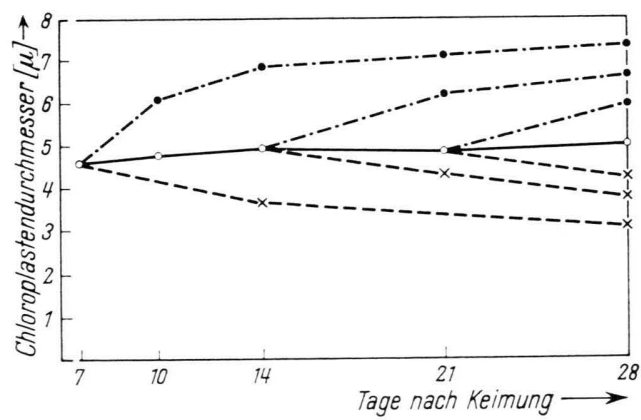

Abb. 2. Die Chloroplastengröße im Dauer-HR sowie im $\mathrm{HR} \rightarrow \mathrm{Bl}-$ und $\mathrm{HR} \rightarrow$ DK-Umsetzversuch. 
von $7,8 \pm 0,32 \mu$ (Abb. 3). Es ergibt sich also, daß unter den hier verwendeten Versuchsbedingungen bei ähnlicher photosynthetischer Leistung (gleiche Quantenstromdichte der Lichtfelder, vgl. MoHr und Ohlenroth $^{1}$ ) die Plastiden der Bl-Vorkeime ca.

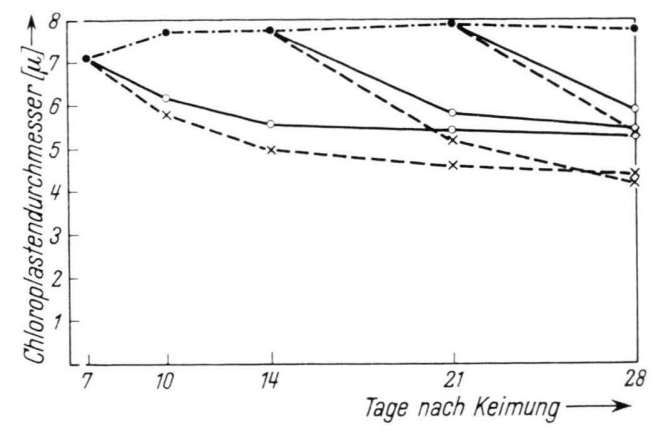

Abb. 3. Die Chloroplastengröße im Bl und ihre Größenreduktion im HR und DK.

1,6-mal größer sind als die der HR-Protonemen. Für diesen Effekt kann demnach nicht die Photosynthese verantwortlich gemacht werden, sondern nur die verschiedene Wirksamkeit der verwendeten Lichtqualitäten im Zusammenhang mit den morphogenetischen Prozessen.

Mit der Größe der Chloroplasten ändert sich auch ihre Zahl pro Zelle. Während im Bl die meisten Chloroplasten gebildet werden, ist ihre Zahl in den HR-Zellen absolut geringer und noch kleiner ist sie in den Zellen der DK-Vorkeime. In den Bl-Zellen liegen die Chloroplasten sehr dicht, so daß vor allem in den Randzonen einzelne Plastiden nicht erkannt werden können. Auf eine Auswertung der Chloroplastenzahl wurde deshalb verzichtet.

b) Die Änderung der Chloroplastengröße im einfachen Umsetzversuch

Das Umsetzen in eine andere Lichtqualität erfolgte 7, 14 oder 21 Tage nach der Keimung und Kultur unter konstanten Lichtbedingungen. Während nach dem Lichtwechsel die Basalzelle ihre Form und Größe beibehält, kann ganz allgemein gesagt werden, daß sich die Chloroplastengröße entsprechend der Wellenlänge des neu einwirkenden Lichtes ändert. Hierbei vergrößern sich die Plastiden der im DK gehaltenen Vorkeime, gleichgültig zu welchem Zeitpunkt sie umgesetzt werden, innerhalb von 7 Tagen stets um den gleichen Wert. Im HR um 1,4 $\mu$ und im Bl um 3,0 $\mu$ (Abb. 1). Hingegen ist die Zunahme der Durchmesser der im HR gebildeten Chloroplasten im $\mathrm{Bl}$ verschieden. Sie beträgt nach 7 Tagen Bl-Einwirkung bei 7 Tage HR-Vorkultur $2,3 \mu$,

bei 14 Tage HR-Vorkultur $1,2 \mu$ und

bei 21 Tage HR-Vorkultur $0,9 \mu$.

Die HR-Plastiden werden offensichtlich gegenüber der Wirkung der Bl-Strahlung stabiler. Im DK schrumpfen die HR-Chloroplasten und nähern sich einem Größenwert, der dem der DK-Plastiden nahekommt.

Bemerkenswert ist, daß die DK-Plastiden sowohl im HR als auch im Bl nie die Größe der im DauerHR oder Dauer-Bl entwickelten Plastiden erreichen. Dasselbe gilt auch für die im HR entstandenen Chloroplasten, die in $\mathrm{Bl}$ gebracht werden. Umgekehrt werden auch die Bl-Chloroplasten im HR oder DK nicht so klein wie die entsprechenden HR- oder Bl-Kontrollen. So schrumpfen sie im HR bis auf einen Durchmesser von 5,3 $\mu$ (HR-Kontrolle 4,8 $\mu$ ) und im DK auf 4,1 $\mu$ (DK-Kontrolle ca. 3,0 $\mu$ ) (Abb. 3). Hierbei ist die Größenreduktion nicht nur auf einen Substanzverlust zurückzuführen, sondern offenbar auch auf Teilungen der Plastiden. Daß trotzdem die Zahl der Plastiden in den Zellen nicht zunimmt, sondern immer weniger wird, kann man damit erklären, daß sich relativ viele sehr stark zurückbilden oder gar degenerieren.

c) Das Verhalten der Chloroplasten in Doppelumsetzversuchen

Die zeitlichen Kombinationen der einzelnen Lichtqualitäten ergeben sich aus den Abb. 4 und 5. Diese Versuche zeigen, daß die Chloroplasten in den Vorkeimen von Dryopteris filix-mas auch bei mehrmaligem Wechsel der Lichtqualität sich auf die jeweils wirkende Wellenlänge einstellen können (vgl. Abschn. a). Aus Abb. 4 geht hervor, daß die HRChloroplasten ihre im $\mathrm{Bl}$ erworbene Größe in einer anschließenden HR-Periode relativ rasch wieder ver-

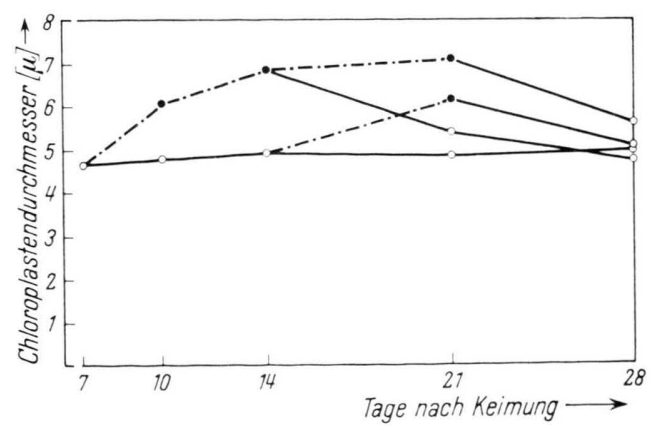

Abb. 4. Die reversible Änderung der Chloroplastengröße im $\mathrm{HR} \rightarrow \mathrm{Bl} \rightarrow$ HR-Doppelumsetzversuch. 
lieren und ihren ursprünglichen Durchmesser wieder erreichen. Dagegen bleiben die DK-Plastiden, nachdem sie von $\mathrm{Bl}$ wieder in DK gesetzt werden, stets größer (um $0,9 \mu$ ) als sie ursprünglich waren (Abb. 5). Es muß also in ihnen während der Bl-

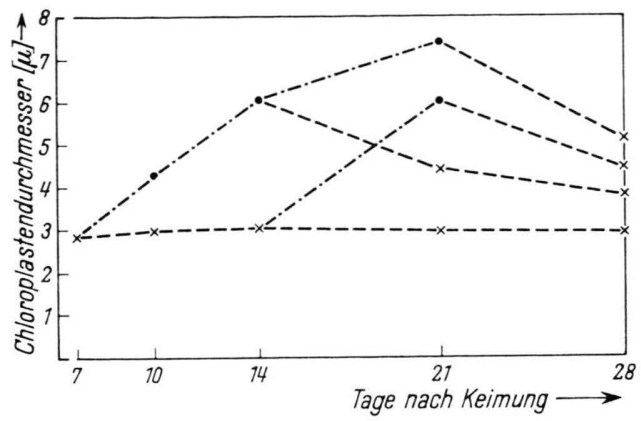

Abb. 5. Die Ausbildung der Chloroplasten im $\mathrm{DK} \rightarrow \mathrm{Bl} \rightarrow$ DK-Doppelumsetzversuch.

Periode eine Substanz gebildet werden, die sie vorher nicht in dem Maße besessen haben und die sie bei einer folgenden Schrumpfung nicht mehr abgeben (vgl. einfachen Umsetzversuch $\mathrm{Bl}-\mathrm{DK}$ ). Nähere Aussagen hierüber müssen weitere Versuche erbringen.

\section{Diskussion}

Die Chloroplasten-Ausbildung bei Dryopteris filix-mas wird durch die photomorphogenetisch wirksame Strahlung kontrolliert. Die Größenunterschiede der im DK, HR bzw. Bl gezogenen Chloroplasten beruhen sicher nicht auf einem verschiedenen Stärkegehalt der Plastiden, zumal die Vorkeime unter den gewählten Bedingungen sowohl im $\mathrm{Bl}$ als auch im HR annähernd die gleiche PhotosyntheseLeistung besitzen (Mohr und OHLENroth ${ }^{1}$ ). Viel eher ist ein Zusammenhang mit der von Нотта und Osawa $1958^{4}$ und Оhlenroth und Mohr ${ }^{2}$ bei der Bildung und dem Wachstum von zweidimensionalen Prothallien gefundenen Erhöhung des Proteingehaltes naheliegend. Brawerman et al. ${ }^{9}$ wiesen an Euglena gracilis nach, daß die bei Belichtung gebildeten Proteine fast ausschließlich in den Chloroplasten gelagert sind. Desgleichen konnten $\mathrm{ZuCKER}_{\mathrm{U}}$

9 G. Brawermann, A. O. Pogo u. E. Chargaff, Biochim. biophysica Acta [Amsterdam] 55, 326 [1962].

10 W. Menke, Z. Bot. 32, 273 [1938].

11 A. T. Jagendorf u. S. G. Wildmann, Plant Physiol. 29, 270 [1954]. und Stinson JR. ${ }^{6}$ bei ihren Untersuchungen an Oenotherenbastarden zeigen, daß $75 \%$ aller Blattproteine in den grünen Plastiden liegen. Andere Autoren fanden allerdings einen weniger großen Eiweißgehalt der Plastiden (Menke ${ }^{10}$, Jagendorf und Wildmann ${ }^{11}$ ). Trotzdem zeigen alle diese Befunde deutlich, daß den Chloroplasten innerhalb des Proteinstoffwechsels der Zelle eine bedeutende Rolle zukommt. Wir neigen deshalb dazu, die Zunahme der Chloroplastengröße in unseren Versuchen auf eine Erhöhung des Proteingehaltes derselben zurückzuführen. Im HR oder DK wird das Protein abgebaut oder an das umgehende Plasma abgegeben, was dann eine Größenreduktion zur Folge hat. Daß hierbei die DK-Plastiden im Doppelumsetzversuch in der abschließenden DK-Phase nicht wieder auf ihre ursprüngliche Größe reduziert werden, kann nur auf eine durch das Licht bedingte Synthese einer Substanz zurückgeführt werden, die sie nicht mehr abgeben können. Es ist möglich, daß es sich hierbei um sogenannte Struktureiweiße handelt, die bei einer DK-Kultur in den Plastiden nicht ausgebildet werden.

Parallel mit der Änderung der Plastidengröße geht auch eine strukturelle Veränderung derselben. MühLETHALER und $F_{\text {REY }}-W_{\text {YsSLing }}{ }^{12}$ zeigten, da $\beta$ lamellare Strukturen der Plastiden nur bei Belichtung entstehen; ABEL ${ }^{13}$ konnte eine Wechselwirkung von Granazahl, Granagröße und Chloroplastengröße in Abhängigkeit von der Lichtintensität aufweisen. Im Rahmen unserer Untersuchungen wurden jedoch Änderungen in diesen Strukturen bisher nicht quantitativ verfolgt.

Diese Phänomene der Chloroplasten-Vergrößerung bzw. -Schrumpfung in Abhängigkeit von der Lichtqualität und Lichtintensität sind im Prinzip schon lange bekannt. SENN ${ }^{14}$ stellte bei den Chloroplasten einiger Phanerogamen und Kryptogamen eine "weitgehende Plastizität" fest, die ihnen erlaubt, auf bestimmte Einflüsse, besonders Licht, mit einer Gestaltsveränderung zu reagieren, wobei dieser Vorgang reversibel ist. Die vorliegende Arbeit zeigt, daß Größe und wohl auch Struktur der Chloroplasten über photochemische Reaktionen, die nichts mit der Photosynthese zu tun haben, gesteuert werden können.

12 K. Mühlethaler u. A. Frey-Wyssling, J. biophysic biochem. Cytol. 6, 507 [1959].

13 B. Abel, Z. Bot. 50, 60 [1962].

14 G. Senn, Gestalts- und Lageveränderung der Chromatophoren, Leipzig, Wilhelm-Engelmann-Verlag, 1908. 